\title{
Simulation of multiwave pumped fiber Raman amplifiers
}

\author{
G.S. Felinskyi ${ }^{1}$, P.A. Korotkov ${ }^{2}$

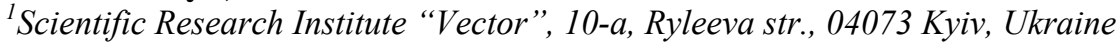 \\ Phone: +380-44-468-3289, fax:+380-44-468-1210; e-mail:felinsky@ndivektor.kiev.ua \\ ${ }^{2}$ Taras Shevchenko Kyiv National University, 2, Academician Glushkov prospect, 03127 Kyiv, Ukraine \\ Phone: +380-44-526-0570, fax:+380-44-526-0531; e-mail: pak@mail.univ.kiev.ua
}

\begin{abstract}
The design model for development of optical fiber Raman amplifiers with the multiwavelength pumping scheme is proposed in this work. Our simulation based on the oscillator theory and spectroscopic model for the analysis of the Raman gain spectrum. Introducing the oscillatory lineshape functions gives the possibility for simple describing the wavelength dependence of Raman gain in optical fibers and it is very useful for the estimation of the gain bandwidth, Raman laser conditions, noise perfomance, and amplification processes in the Raman amplifiers. Modeling results are obtained without traditional solving the complicated system of coupled equations. The concept of an actual band based on the lineshape function is useful for the design of fiber Raman amplifiers with multiple wavelength pumping. Model validity is confirmed by the experimental measurements of amplified spontaneous emission in silica fiber. Proposed modeling allows us to analyze the fiber Raman amplifier with combined multiwavelength pumping source for the extension of amplification bandwidth to L-band, which has the broad bandwidth over $80 \mathrm{~nm}$ and low gain ripple less than $0.5 \mathrm{~dB}$. The proposed spectroscopic model can be further extended for the analysis of the complex spontaneous Raman scattering spectra with other doping materials.
\end{abstract}

Keywords: optical fiber amplifier, optical fiber communication, fiber Raman amplifier.

Manuscript received 26.06.06; accepted for publication 23.10.06.

\section{Introduction}

Fiber Raman amplifiers (FRA) are becoming more and more essential in wavelength-division-multiplexing optical communication systems with terabit capacity and are being deployed in almost every new long-haul and ultralong-haul fiber-optic transmission systems [1-3].

The fiber Raman amplifier with multiple pump waves (MW-FRA) can help overcome bandwidth of $\mathrm{C}+\mathrm{L}$ band $[3,4]$. The multiple-wave pumping enables to expand the gain bandwidths to the theoretical limit of approximately up to $12 \mathrm{THz}$ [3]. Gain bandwidths of MW-FRAs are limited by the requirement that the pump and signal channels should occupy separate wavelength regions. Thus the theoretical limit is determined by the fact that the Raman gain profile in fused silica fibers possesses peak shift approximately $13 \mathrm{THz}\left(440 \mathrm{~cm}^{-1}\right)$.

The advantages of fiber Raman amplifiers over the optical amplifiers include the possibility to operate in any wavelength region and superior noise performance of distributed amplification, as well as permits, with the appropriate choice of pump wavelengths and powers, flattening of the gain profile over the whole bandwidth [4].

Despite the simplicity of the architecture of a Raman amplifier, it is not simple to obtain the gain profile of FRA with the multiple wavelength-pumping schemes by using the coupled equations directly. Many factors in the design of amplifiers such as pump-to-pump power transfer, signal-to-signal power transfer, pump depletion (saturation), double Rayleigh scattering (multipath interference), and amplifier spontaneous noise should be considered [4]. In the distributed optical fiber Raman amplifier, because of an interaction between the pumps and the amplified signals the pump depletion is usually described by the well-known set of coupled equations $[4-5,10]$. The interaction between the pumps and the strength of the coupling between a pump wave and a signal wave due to the stimulated Raman scattering (SRS) are determined by the Raman gain coefficient. It is a fundamental parameter for the Raman amplification.

The simplistic design of the multiple pumping Raman amplifier results in a very poor amplifier. In the 
previous report [4], evenly spaced eight pumps with equal pump power of $120 \mathrm{~mW}$ were used for FRA with the broad gain bandwidth. The gain variation, however, was more than $10 \mathrm{~dB}$. In order to achieve the broad gain bandwidth with the small gain ripple, the power and wavelength of each pump diode should be carefully chosen.

Thus, the parameters optimization of FRA with multiwavelength pumping scheme is a subject of high interest now but the presented methods almost exclusively directly based on the too complicated system of coupled equations for the SRS process. Numerical optimization methods for the optimal determination of the power and wavelength of each pump source based on the coupled equations such as simulated annealing algorithm [5], single-layer feed forward neural networks [6], and so on [7] have been investigated. In the previous methods, however, when the pump wavelengths are automatically arranged and based only on the peak Raman gain wavelength, one may result to practically undesirable pump power distributions due to an inadequate choice of frequency dependent factors like the Raman gain coefficient and effective area in the coupled equations. All the parameters of the Raman amplification can be obtained from propagation equations for signal and pump waves only in the case with a proper theoretical model of the Raman gain profile in optical fibers. The theoretical analysis of the Raman gain coefficient with one pumping wavelength in optical fiber and comprehensive analysis of its scaling with wavelength, modal overlap, and material composition are also reported [8].

In this work, we will propose the spectroscopic model for the analysis of Raman gain spectrum based on the oscillator theory and its application to the design of optical FRA with the multiwavelength pumping scheme. By introducing the oscillatory lineshape function of $S_{\mathrm{R}}(v)$, we can analyze the amplification process of FRA. Main advantage of our design model is that a lot of important FRA parameters can be obtained without too complicated solving the coupled equations. The experimental configuration of FRA with the wide gain bandwidth and small gain ripple will be proposed and analyzed using the actual band model based on $S_{\mathrm{R}}(v)$. The threshold pumping power and effective noise figure for FRA can be analyzed using the proposed model that is also applicable for versatile fibers with other doping materials.

\section{Theoretical background of spectroscopic model for FRA design}

The Raman gain coefficient determines the strength of the coupling between a pump beam and a signal beam due to the stimulated Raman scattering. In the theoretical model for the Raman amplification process in optical single-mode fibers basically used are two approaches, namely, quantum and semiclassical. Using a combination of these two approaches it was demons- trated how the fiber temperature affects the performance of the Raman amplifier and how the Raman gain coefficient scales with wavelength [8].

The quantum mechanical description of the interaction between optical fields and molecular phonons is used to derive rate equations for the generation and amplification of the Stokes photons. The rate equations immediately describe how the fiber temperature affects amplifier performance.

The semiclassical model makes it possible to evaluate the Raman gain coefficient of an optical fiber. From the Maxwell equations for classical optical fields and a quantum description of molecular motion as damped harmonic oscillator, one can obtained an expression for the Raman gain coefficient, including its dependence on material and field properties. It gives a connection to the traditional concept of nonlinear susceptibility. In addition, the theory provides the scaling of the gain coefficient with the wavelength and spatial overlapping of the pump and signal modes.

In the Raman process when the pumping frequency is $\omega_{p}$, the scattering molecule may be described as a quantum oscillator with frequency $\omega_{v}$, and the rate equation for change per unit length $z$ of the Stokes scattered photon number $n_{s}$ at $\omega_{\mathrm{S}}=\omega_{p}-\omega_{v}$ is [8]

$$
\begin{aligned}
& \frac{d n_{\mathrm{S}}}{d z}=C \rho\left(\hbar \omega_{f}\right) \frac{\omega_{p} \omega_{\mathrm{S}}}{\omega_{v}} \times \\
& \times\left\{n_{\mathrm{S}}\left(n_{v}+1\right) n_{p}-n_{\mathrm{S}} n_{v} n_{p}-n_{\mathrm{S}} n_{v}+\left(n_{v}+1\right) n_{p}\right\},
\end{aligned}
$$

where

$$
C=\left|\partial \alpha_{i j} / \partial q_{k}\right|^{2}\left(\pi \hbar^{2}\right) /\left(4 V^{2} N m \varepsilon_{\mathrm{S}} \varepsilon_{p} v\right),
$$

$\partial \alpha_{i j} / \partial q_{k}$ is the differential polarizability; $\vec{q}$ is displacement vector corresponding to $i$-th component of the dipole moment; $\hbar=h / 2 \pi$ is Plank's constant; $N$ is the number of oscillators in the interaction volume $V ; \varepsilon_{\mathrm{S}}$, $\varepsilon_{p}$ are the dielectric constants for the pumping and Stokes waves, respectively; $v$ is the phase velocity of the Stokes wave.

The density of states $\rho\left(\hbar \omega_{f}\right)$ depends on the damping involved in the molecular transition from the final state to the initial one. There are many processes that restore the system to the thermal equilibrium. Often the Lorentzian line shape is used for $\rho\left(\hbar \omega_{f}\right)[8,11]$.

Since the phonons are assumed to be in equilibrium at a temperature $T$, the occupation number $n_{v}$ is the thermal equilibrium number $n_{v}=\left[\exp \left(\hbar \omega_{v} / k_{\mathrm{B}} T\right)-1\right]^{-1}$, where $k_{\mathrm{B}}$ is the Boltzmann constant.

The rate equation (1) for the Stokes scattered photons consists of four terms that are often referred to as the stimulated emission, stimulated absorption, spontaneous absorption, and spontaneous emission, respectively. It should be noted that the spontaneous emission terms are proportional to $n_{v}+1$ for the Stokes photon and $n_{v}$ for the anti-Stokes one and thus depends on the temperature of the fiber. 
On the other hand, the difference in stimulated emission and absorption terms does not depend on the phonon number $n_{v}$ and therefore is temperatureindependent. In addition, the spontaneous absorption term is typically much smaller than the other terms and therefore can be neglected.

The total amplified power over all signal band of the optical fiber Raman amplifier with one pumping source [9] is $P_{\text {sig }}(z)=\int d v p_{\text {sig }}(v, z)$, where $v$ is the Stokes frequency, and the signal spectral power density $p_{\text {sig }}(v, z)$ is the Stokes power $p_{\mathrm{S}}(v, z)$ in $z$-point along the fiber per unit frequency range. When the pumping saturation is negligible,

$$
p_{\text {sig }}(v, z)=p_{\mathrm{S}}(v, 0) \exp \left[-\alpha z+\sigma(v)\left(1-e^{-\alpha z}\right)\right],
$$

where $\alpha$ is the average fiber attenuation in the signal band and $\sigma(v)=\sigma_{0} S(v)$. The dimensionless function $S(v)$ is the lineshape function normalized so that its peak value is unity, and $\sigma_{0}=g_{\mathrm{R} \max } P_{0} /\left(A_{\mathrm{eff}} \alpha\right)$, where $g_{\mathrm{Rmax}}$ is the peak Raman Stokes gain coefficient, $P_{0}$ is the input pump power, and $A_{\text {eff }}$ is the effective area of the pump beam.

The stimulated Raman scattering (SRS) process has the Stokes shifted frequencies within the line of spontaneous scattering by vibration modes of fiber material. According to the semiclassical derivation [8], SRS can be described in the terms of classical optical fields and the molecular system can be treated as a forced harmonic oscillator. This model reveals the connection between the differential polarizability $\left(\partial \alpha_{i j} / \partial q_{n}\right)$ of a molecular lattice and complex value of its nonlinear susceptibility $\chi^{(3)}$, a quantity traditionally used in nonlinear optics.

$$
\chi_{i j k l}^{(3)}(\omega)=\frac{N}{12 m \varepsilon_{0} V} \frac{1}{\omega_{v}^{2}-\omega^{2}+i \omega \Gamma} \cdot \sum_{n} \frac{\partial \alpha_{i j}}{\partial q_{n}}\left(\frac{\partial \alpha_{k l}}{\partial q_{n}}\right),
$$

where $\omega_{v}$ is the undamped resonance phonon frequency, $\omega$ is the angular frequency of a phonon, $\Gamma$ is the phonon damping constant, $q_{k}$ is the coordinate that describes the local displacement that results from the time-dependent electric field, $m$ is the mass associated with the vibration, $N$ is the number of oscillators in the interaction volume $V, \varepsilon_{0}$ is the vacuum permittivity.

Since an optical fiber is made of a silica glass, which is an amorphous material, the fourth-rank tensor $\chi^{(3)}$ is isotropic. Thus, $\chi^{(3)}$ has only 21 nonzero elements, the indexes of which are either identical to $\chi_{i i i i}^{(3)}$ or occur in pairs $\chi_{i j j i}^{(3)}, \chi_{i j i j}^{(3)}$, and $\chi_{i j j j}^{(3)}$. Therefore, the Raman gain coefficient at the Stokes frequency $\omega_{\mathrm{S}}=$ $\omega_{p}-\omega_{v}$ can be written as [8]

$g_{\mathrm{R}}(\omega)=-\frac{3 \omega_{\mathrm{S}}}{\varepsilon_{0} c^{2} n_{p} n_{\mathrm{S}}} \frac{\operatorname{Im}\left[\chi_{i i i i}^{(3)}(\omega)+\chi_{i j j i}^{(3)}(\omega)\right]}{2 A_{\mathrm{eff}}^{p \mathrm{~S}}}$.
Assuming that the third-order susceptibilities are frequency-independent, we can note that the gain coefficient $g_{R}$ linearly depends on the frequency of the Stokes-shifted wave, and its frequency dependence can be described by the imaginary part of nonlinear susceptibility $\chi^{(3)}$ such as the resonant denominator of phonon harmonic oscillator (see Eq. (3)).

Pumping saturation is not negligible in distributed optical fiber Raman amplifier with multiple wavelengths pumping. The pumps depletion caused by interaction between the pumps and the amplified signals is described by the well-known set of coupled equations [4-6, 8].

Different forms of these equations are usually applied to modeling the Raman amplification with multiple pumps. Equations for the multiwavelength pumping scheme, which preserves the photon number and include the fiber loss $\alpha(v, T)$ and Rayleigh backscattering $\gamma_{b}(v)$, can be combined to form one general equation [7], in which multiple channels with optical bandwidths $\Delta v$ are included in the codirection (superscript "+") and counterdirection (“-”):

$$
\begin{aligned}
& \pm \frac{d P_{i}^{ \pm}}{d z}=-\alpha\left(v_{i}, T\right) P_{i}^{ \pm}+\gamma_{b}\left(v_{i}\right) P_{i}^{\mp}+ \\
& +P_{i}^{ \pm} \sum_{j}^{v_{j}>v_{i}} \frac{g_{\mathrm{R}}\left(v_{j}, v_{i}\right)}{A_{\mathrm{eff}}\left(v_{j}, v_{i}\right)}\left(P_{j}^{+}+P_{j}^{-}\right) \Delta v+ \\
& +2 \sum_{j}^{v_{j}>v_{i}}\left(P_{j}^{+}+P_{j}^{-}\right) h v_{i} \Delta v \frac{g_{\mathrm{R}}\left(v_{j}, v_{i}\right)}{A_{\mathrm{eff}}\left(v_{j}, v_{i}\right)}\left[n_{\mathrm{B}}\left(v_{j}-v_{i}\right)+1\right]- \\
& -P_{i}^{ \pm} \sum_{j}^{v_{j}<v_{i}} \frac{V_{j}}{V_{i}} \frac{v_{i}}{v_{j}} \frac{g_{\mathrm{R}}\left(v_{i}, v_{j}\right)}{A_{\mathrm{eff}}\left(v_{i}, v_{j}\right)}\left(P_{j}^{+}+P_{j}^{-}\right) \Delta v+ \\
& +2 \sum_{j}^{v_{j}<v_{i}}\left(P_{j}^{+}+P_{j}^{-}\right) h v_{i} \Delta v \frac{v_{i}}{v_{j}} \frac{V_{j}}{V_{i}} \frac{g_{\mathrm{R}}\left(v_{i}, v_{j}\right)}{A_{\mathrm{eff}}\left(v_{i}, v_{j}\right)} n_{\mathrm{B}}\left(v_{i}-v_{j}\right),
\end{aligned}
$$

where the subscript $i$ represents the $i$-th wavelength with the frequency $v_{i}$ and $V_{i}, P_{i}$ are the group velocity and optical powers, respectively. $A_{\text {eff }}(v)$ is the fiber effective area and $g_{\mathrm{R}}\left(v_{i}, v_{j}\right)$ is the Raman gain coefficient at the signal frequency $\left(v_{j}\right)$ and pumping frequency $\left(v_{i}\right) ; n_{\mathrm{B}}(v)$ is the phonon Bose-Einstein distribution.

The term in (5) with $n_{\mathrm{B}}+1$ factor describes the Stokes component of amplified spontaneous emission (ASE) generation, and the term with $n_{\mathrm{B}}$ factor describes anti-Stokes ASE generation, which are the result of photon number conservation.

In general, the frequency dependence of the Raman gain coefficient $g_{\mathrm{R}}(v) \sim S(v)$ of each pumping wavelength is determined by lineshape function of $S(v)$ in accordance with the stimulated Raman scattering process. The Raman gain coefficient is a fundamental parameter for the Raman amplification. 
When the frequency dependence of the Raman gain $g_{\mathrm{R}}(v)$ is modeled as a frequency independent constant or simple single peak, for example as a triangle, the numerical methods may give the unsatisfactory results because the solutions of coupled equation (5) are very sensitive to absolute values of equation coefficients. In the cases of inaccuracy determination of $g_{\mathrm{R}}\left(v_{i}, v_{j}\right)$ in equation (5), the numerical methods usually can not be capable of fully handling FRA optimization problem. It is necessary to take into account the gain spectra in fibers with the best possible accuracy for the Raman amplifier system. In other hand, as we shall show below, the spectroscopic modeling of gain spectrum gives the possibility to determine the FRA bandwidth without complicated solving the coupled wave equations.

\section{Actual band model}

More often the frequency distribution of the Raman cross-section is described by two types of lineshape functions $S(v)$ in spectroscopy. First, it is the Lorentzian lineshape $S_{\mathrm{L}}(v)[9,11]$.

$$
S_{\mathrm{L}}(v)=\frac{(w / 2)^{2}}{\left(v-v_{0}\right)^{2}+(w / 2)^{2}},
$$

where $v_{0}$ is the center frequency and $w$ is the full width at half maximum (FWHM). This function is widely used for many spectroscopic models, particularly for the Raman scattering.

From the equations (3) and (4), the frequency dependence $S(v)$ of the Raman gain can be written as

$$
S(v)=-\operatorname{Im}\left[\frac{1}{v_{0}^{2}-v^{2}+i v \gamma}\right]=\frac{v \gamma}{\left(v_{0}^{2}-v^{2}\right)^{2}+v^{2} \gamma^{2}},
$$

where $v$ is the wave numbers $\left(v_{0}=\omega_{v} / 2 \pi c\right.$, $v=\omega / 2 \pi c$, and $\gamma=\Gamma / \pi c)$ and $\gamma$ is approximately FWHM of $S(v)$. The normalized function of $S_{\mathrm{R}}(v)$ is the lineshape one, it is dimensionless, and can be written as

$$
\begin{aligned}
& S_{\mathrm{R}}(v)=\gamma v_{0} S(v), \\
& S_{\mathrm{R}}\left(v_{0}\right)=1 .
\end{aligned}
$$

Since the Stokes shifted line profile of the Raman gain in SRS process repeated the line shape of phonon vibrations in fiber material, as seen from (3) and (4), the lineshape functions $S_{\mathrm{L}}(v)$ and $S_{\mathrm{R}}(v)$ can be applied for the modeling the Raman amplification. The Lorentzian lineshape function $S_{\mathrm{L}}(v)$ is very closed to the normalized curve $S_{\mathrm{R}}(v)$ for the phonon harmonic oscillator in almost all meaning region for the same parameters, especially, when $v_{0} \gg>, \gamma$, as shown in Fig. 1 . The function $S_{\mathrm{R}}(v)$ for a phonon harmonic oscillator with dumping is very often miscalled as the Lorentzian function. It is incorrect, in common words, because historically the function (6) was named as the Lorentzian, and it differs from the function $S_{\mathrm{R}}(v)$ for the phonon harmonic

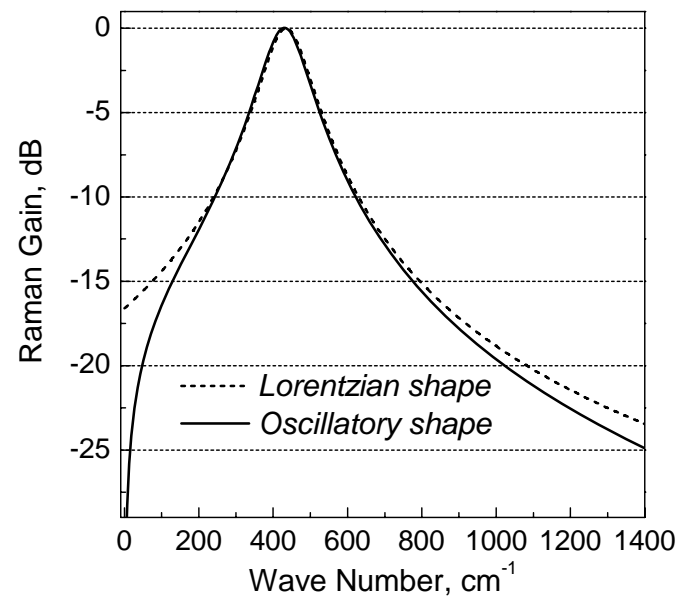

Fig. 1. Lineshape functions $S_{\mathrm{L}}(v)$ (dotted line) and $S_{\mathrm{R}}(v)$ (solid line) with $v_{0}=435 \mathrm{~cm}^{-1}, w / 2=\gamma=130 \mathrm{~cm}^{-1}$ are shown in the logarithmic scale.

oscillator (7), as one can see in Fig. 1. A small shift at the line center frequency of $S_{\mathrm{R}}(v)$ in comparison with $S_{\mathrm{L}}(v)$ deals with the influence of dumping shift on the phonon harmonic oscillator. The extent of the shift increases with $\gamma$ because the maximum value of the Lorentzian function is equal to unity $\left(S_{\mathrm{L}}\left(v_{0}\right)=1\right)$ and independent of $\gamma$ at $v_{0}$. Larger difference is visible in the low frequency region as shown in Fig. 1. In contrast with the oscillatory function $\left(S_{\mathrm{R}}(0)=0\right)$, the Lorentzian function is not zero at $v=0$.

A small difference between two curves of $S_{\mathrm{L}}(v)$ and $S_{\mathrm{R}}(v)$ takes place in the long-wave region. The integral intensity of both line shapes, however, remains almost the same at $v_{0}>>w, \gamma$. We use the oscillatory function $S_{\mathrm{R}}(v)$ in our modeling in accordance with the conventional Raman gain theory.

Figs $2 \mathrm{a}$ and $\mathrm{b}$ show the measured results of the Stokes Raman line with $20 \mathrm{~mol} . \% \mathrm{GeO}_{2}$ [11] and the theoretical results based on the proposed lineshape function $S_{\mathrm{R}}(v)$ (dotted line in Fig. 2b), respectively. The phonon harmonic oscillator function $S_{\mathrm{R}}(v)$ when the undamped resonant phonon frequency $\left(v_{0}\right)$ and photon damping constant $(\gamma)$ are 435 and $130 \mathrm{~cm}^{-1}$, respectively, is the best fitting curve to experimental data of the frequency dependence of the Raman gain in highly Gedoped fibers as seen in Fig. $2 b$.

To estimate the fitting accuracy, we used the square-law deviation $\sigma^{2}=\left[S_{\mathrm{R}}(v)-S_{\exp }(v)\right]^{2} / S_{\exp }^{2}\left(v_{0}\right)$ for variation of the oscillator lineshape function of $S_{R}(v)$ from the experimental gain profile $S_{\exp }(v)$, which shown in Fig. 2b. It should be noted that $\sigma^{2}<1 \%$ for all wave numbers within the range from 100 up to $600 \mathrm{~cm}^{-1}$, and its average value was $\sigma^{2}=0.2 \%$. In addition, the good conformity of the proposed modeling results with the experimental data could be verified by the fact that the difference of the integrated intensity of both gain profiles was less than by $0.8 \%$ in the specified amplification band. 

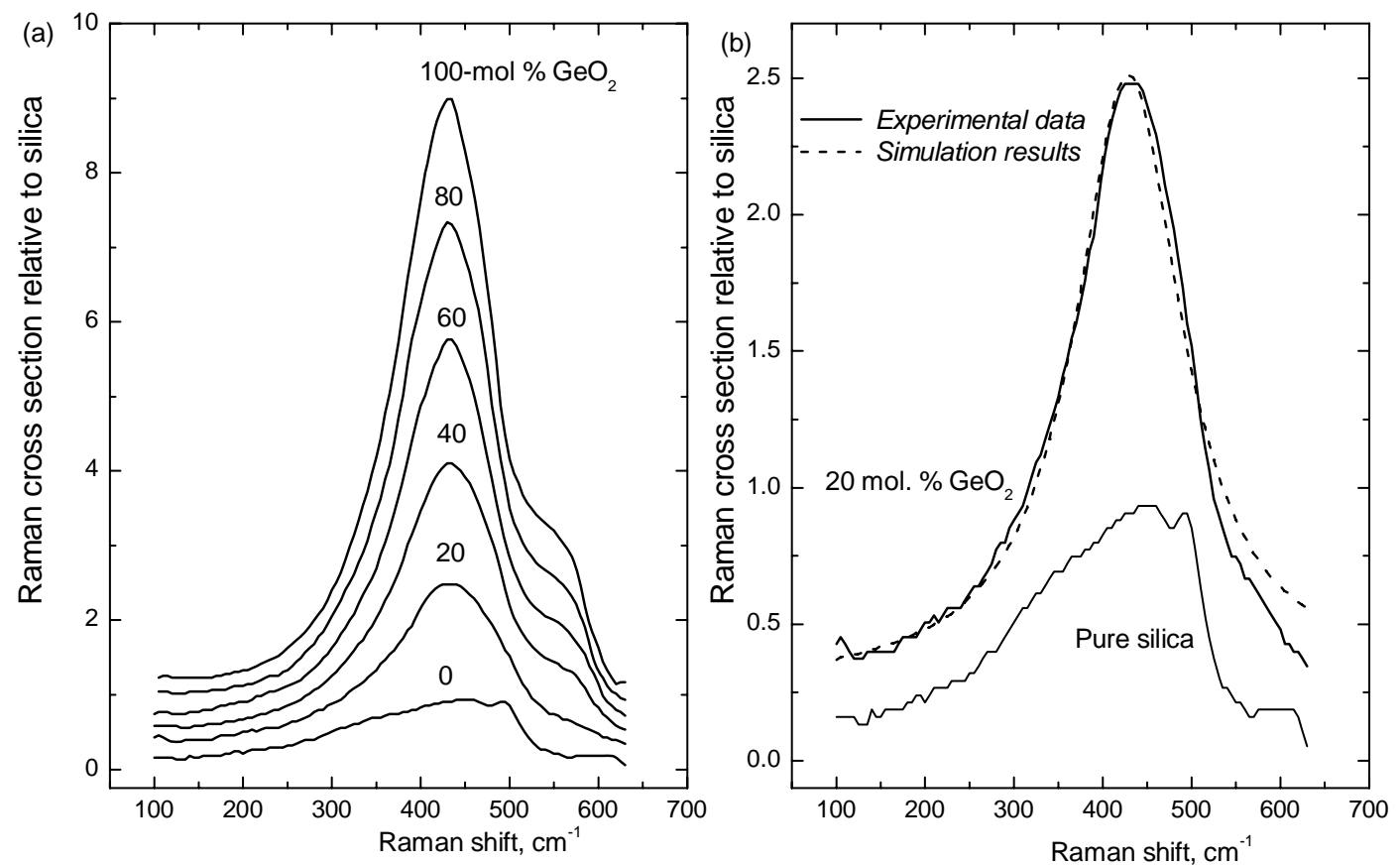

Fig. 2. a - experimental measurements of zero Kelvin Raman cross-section relative to silica in germanosilicate glass [11] (solid lines); $\mathrm{b}$ - approximation of the Stokes Raman line in $20 \mathrm{~mol} . \% \mathrm{GeO}_{2}$ by $S_{\mathrm{R}}(v)$ function (dotted line).

We applied the theoretical results to modeling the amplification process in the Raman amplifiers. The model is based on the analysis of the Raman gain spectrum and allocation of the oscillatory modes, which is involved in SRS process. For each doping material with one dominant vibration mode, it is necessary to get two parameters like the central vibration frequency and its dumping constant in the lineshape function $S_{\mathrm{R}}(v)$. In many cases, it is expedient to use one actual band for the effective account and to display several spectral bands to analyze the complex spectra with many overlapped lines. The real multiple spectra can be represented by one spectral component, which gives the maximal spectrum profile and integrated intensity approximation. Resulting component with proper lineshape $S_{\mathrm{R}}(v)$ we designate as an actual band for a real spectrum. The concept of an actual band is frequently used in spectroscopy. Our expectations about actual band model usefulness for analysis and synthesis of the Raman gain spectra with multiwavelength pumping scheme of high degree was justified by the following simulation results. The actual band approximation of the Raman gain spectrum gives a good fitting curve in Ge-doped fibers as seen in Fig. 2, but conformity is not good for the case of pure silica fibers.

\section{Simulation model approbation}

Based on the actual band model in the Raman gain, we obtain the amplified stimulated emission (ASE) spectra to compare with the experimental results of the effective noise figure with respect to the wavelength [13]. Fig. 3 shows the theoretical results of ASE spectra (solid line) and published data for 6 wavelengths pumping Raman amplifier which covered both $\mathrm{C}+\mathrm{L}$ bands. We compared our simulation results with experimental data for the effective noise figure [13] (points with dashed lines in Fig. 3). Six wavelengths $(1428,1445,1466,1480,1494$ and $1508 \mathrm{~nm}$ with pumping powers of $338,215,83,30$, 19 , and $39 \mathrm{~mW}$, respectively) were used in the experiment [13]. These wavelengths were chosen to uniformly distribute the pumps in optical frequency. Gain flattening is achieved by experimentally tuning of the pump power so the longer wavelength spectral components operating at low powers, just above the lasing threshold.

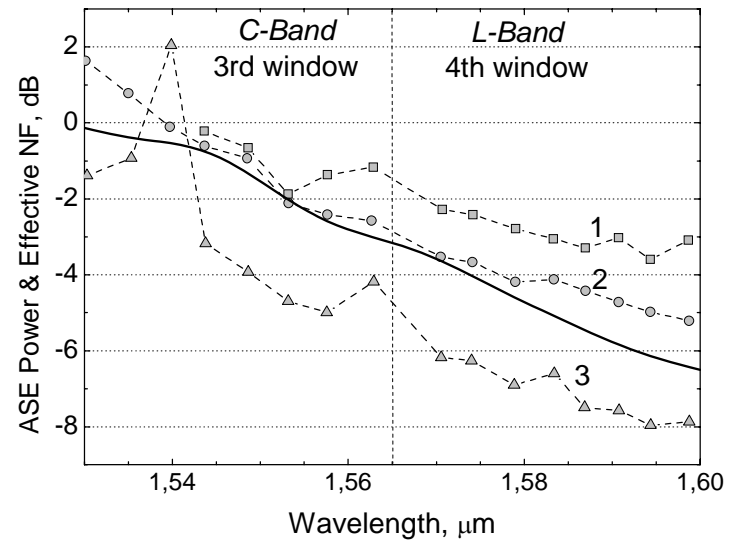

Fig. 3. Simulated ASE spectra (solid line) and measured effective noise figures for 6 wavelengths pumping Raman amplifier in $\mathrm{C}+\mathrm{L}$ band. $1-60 ; 2-100 ; 3-140 \mathrm{~km}$ (Exp. data from [13]). 
Modeling conditions are as follows. The ASE spectral density at FRA output should have the same form as the Raman gain profile with uniform distribution of optical noise. We have modeled the gain profile band based on the pumping wavelengths and pumping powers data as given in Ref. [13]. ASE results in the frequency dependence of the amplifier noise figure (NF) over FRA bandwidth. The shape of NF is proportional to the ASE distribution at the FRA output in experimental conditions of Ref. [13], when gain ripple is minimal. Good conformity of the ASE as a function of the wavelength in comparison with experimental results of NF in FRA could be obtained as seen in Fig. 3. The slope of ASE distribution was $\sim 7 \mathrm{~dB}$ in $\mathrm{C}+\mathrm{L}$-bands. This value was in a good agreement with NF slopes (approximately $7 \mathrm{~dB}$ ) in all the fiber lengths of 60, 100, and $140 \mathrm{~km}$ as seen in Fig. 3 .

The experimental data shown in Fig. 3 were obtained using the distributed Raman amplifier on TrueWave RS optical fiber [13]. However, good conformity of the calculated curves courses for the amplified spontaneous emission with noise figures of the real amplifier specifies applicability of the actual band model for estimation of the Raman amplifier noise performance and realization of engineering calculations.

Abovementioned analysis allows us to make several qualitative conclusions about FRA noise performance. Apparently, the main part of FRA noise is formed in the fiber near to pump sources, because the pumping power is maximal in it. General pumping losses caused by propagation through the fiber including the pump depletion reduces the pumping power that becomes insufficient for the ASE generation. Thus, the average slopes of NF keeps constant regardless of the various fiber lengths, and finally they are the same as for the ASE distribution in Fig. 3.

This conclusion is confirmed by our experimental ASE measurements. We have observed ASE from the single mode fiber span in counterdirection to pump using commercial FRA unit with four LD pump sources (1426, $1436,1456$, and $1466 \mathrm{~nm})$. The maximum pump power was $300 \mathrm{~mW}$ per each LD. The experimental setup is shown in Fig. 4. Output pump power from each LD through the pump combiner and circulator is directed to the $50 \mathrm{~km}$ span of standard single mode fiber. Pumping source allows independently fix the output power of each LD in the range from 0 to $300 \mathrm{~mW}$ using a digital control unit. ASE power from the fiber after circulator is registered by optical spectrum analyzer (OSA). Spectral resolution of OSA was set to $1 \mathrm{~nm}$ for all ASE measurements.

Fig. 5 shows the Stokes ASE power densities in $\mathrm{nW} / \mathrm{nm}$ generated by separate LD pump as a function of the wavelength. Only one LD was active (the rest LDs are turned off) at individual spectra recording and the set of its power levels was: 100, 150, 200, 250, and $300 \mathrm{~mW}$ for each LD wavelength. Wavelength range is normalized to $1440-1650 \mathrm{~nm}$ for 1426 and $1436 \mathrm{~nm}$

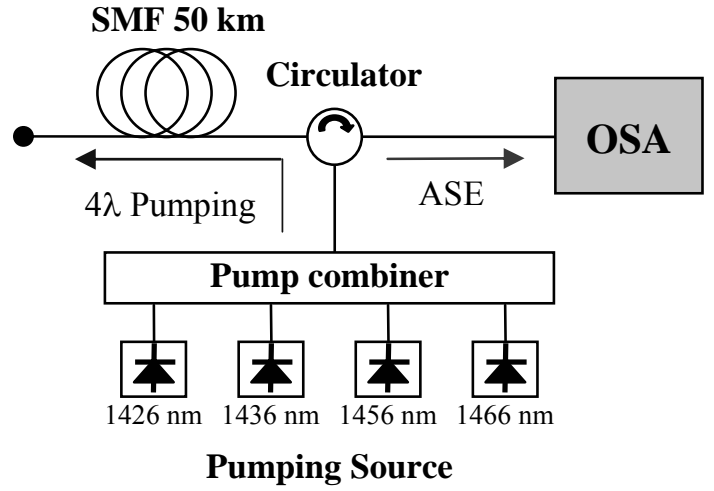

Fig. 4. Experimental setup for ASE measurements with 4 pumping sources.

LDs and wavelength range is shown from 1470 to $1700 \mathrm{~nm}$ for 1456 and $1466 \mathrm{~nm}$ LDs in Fig. 5.

The general view of the spectra submitted in Fig. 5 has strongly pronounced features in a qualitative interpretation of the well-known spectrum of the spontaneous Raman scattering of light in the silica fibers, looking like non-uniform continuum in the Stokes shift range from 0 up to $1400 \mathrm{~cm}^{-1}$. The main difference between the spontaneous Raman spectrum and SRS spectral profile is shown as the raised intensity of frequency components with the small Stokes shifts approximately up to $200 \mathrm{~cm}^{-1}$. Distinction between the spontaneous and stimulated Raman spectra is directly described by the quantum dynamic equation (1). In this equation, the term corresponding to the spontaneous Raman scattering contains the phonon density factor as $n_{\mathrm{B}}(\omega)+1$ that considerably exceeds unity at $T=300 \mathrm{~K}$ in the frequency region less than $200 \mathrm{~cm}^{-1}$, and it infinitely grows when the frequency tends to zero. As it was already noted in Section 2, the SRS process does not depend on the phonon density-of-states alternatively to the spontaneous Raman scattering, and accordingly it does not depend on the temperature. This fact defines the difference of an observable Raman gain spectrum from the measured spontaneous Raman spectrum which is the most appreciable in the small Stokes shift region.

Other feature of the spontaneous Raman scattering is its linearity by nature and thus it does not depend on pump intensity. Thus, the spontaneous Raman crosssection remains constant for each studied material, and its numerical value is determined by physical parameters of substance. The presented ASE measurements show that the absolute power cross-section of the Stokes radiation for each of four pump wavelengths varies from $(2.75 \pm 0.08) \cdot 10^{-6}$, when the input LD pump power is $100 \mathrm{~mW}$, up to $(4.3 \pm 0.2) \cdot 10^{-6}$ for the pump power of $300 \mathrm{~mW}$. The obtained numerical values for ASE cross-section of the order size $\sim 10^{-6}$, correspond to the quantum efficiency of the spontaneous Raman scattering, but it does not SRS because its quantum efficiency should be higher by approximately 


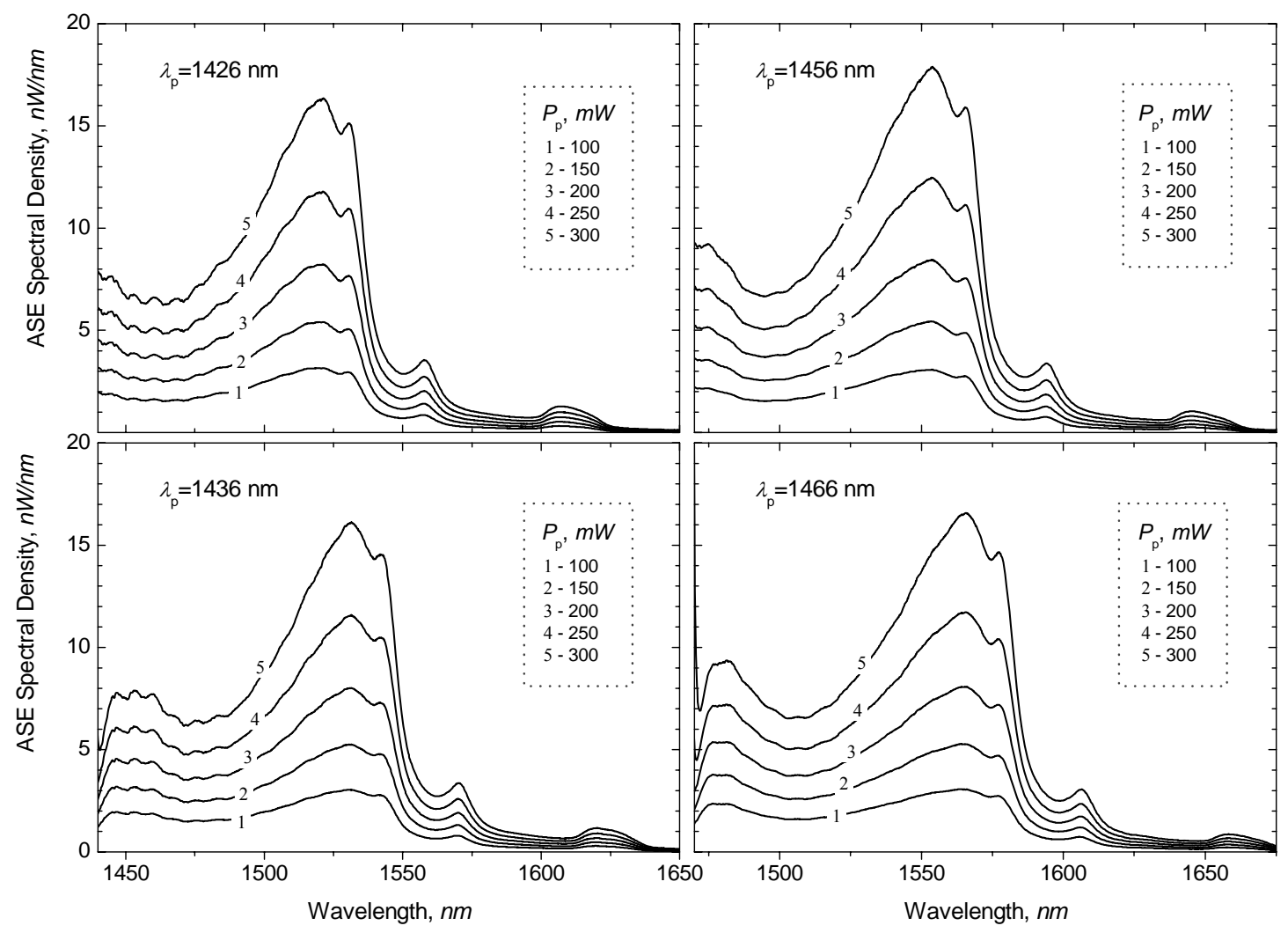

Fig. 5. Stokes ASE powers that are generated with separate pumping sources.

4-5 orders. The absolute ASE cross-section was experimentally determined as the ratio of the total power for the Stokes spectrum integrated over the shifted frequency range from 10 up to $1400 \mathrm{~cm}^{-1}$ to the input pump power.

One can see that the power gain of spontaneous optical noise in the single mode silica fiber is in the limits bounded by enough small values. The Raman quantum efficiency grows no more than $\sim 40 \%$, and it corresponds to increase of on/off gain only about $1.9 \mathrm{~dB}$, when the pump powers are varied by 3 times, i.e., from 100 up to $300 \mathrm{~mW}$.

It is possible to explain such situation from the physical viewpoint as follows. The rather weak ASE generation in the studied pump power range is resulted from the absence of coherence for the Stokes photons arising when nonelastic scattering of pump photons by a huge amount of molecular phonon vibrations with various frequencies. Therefore, the Stokes radiation in addition to its random phase distribution appears as distributed in a very wide frequency range. Both these circumstances obstruct to automatic establish the phase matching conditions necessary for coherent accumulation of the Stokes radiation that would provide the Raman gain. In other words, the Raman interaction in the core of the silica based optical fiber results to "spreading" the pump power along the wide spectrum of the Stokes frequencies. As the creation probability of the inphase Stokes photons with equal frequencies inversely depends on the Raman radiation bandwidth as it appears available pump power insufficiently for the effective Raman noise generation. As a result, the spectral power distribution of ASE observed by us looks more likely the spontaneous Raman scattering, instead of SRS.

It should be noted that these results were obtained with no optical signals in the studied single mode fiber piece. With availability of the signal, its coherent power with the spectral density that is considerably higher than the Stokes noise density starts the concurrence for possession of the pump power during the SRS process. The expending of the pump power for signal amplification causes the accelerated pump depletion in the propagation process along the fiber, simultaneously reducing the effective length of power accumulation of the Stokes noise. Therefore, the ASE gain coefficients measured by us in the single mode silica fiber have the greatest possible quantities, and the noise power of real FRA cannot exceed the absolute values resulted in Fig. 5.

The carried out measurements show that the physical principles of formation of the FRA optical noise with the counter pumping almost correspond to the spontaneous Raman properties in the optical fibers based on fused silica. It is the reason for practically repetition of the spectral density of output FRA optical noise by the spontaneous Stokes Raman distribution. On the other hand, it means that for the definition of the power spectrum of output FRA optical noise, it is enough to know pump power distribution at the amplifier input. 
Therefore, our experimental data on the ASE measurements in silica fibers allows us to get information about the nature of the formation mechanism of the noise parameters in the practical FRA. In addition, the actual band model can be used to estimate the absolute power of the spectral density for optical noise in FRAs as well as for the amplifier bandwidth determination.

To make the spectroscopic model applicable for the spectra with any complexity, it is necessary to use the multiple vibrational mode model with an appropriate set of approximating functions for each participating oscillation in the SRS process.

\section{Modeling results and discussion}

The spectroscopic model allows us to estimate the gain bandwidth of FRA simply and directly with the multiply pumping sources. Based on the proposed model, it is easy to obtain the gain bandwidth of FRA without solving the coupled wave equations, and modeling results may be displayed in the convenient graphic form.

At first, we applied the proposed spectroscopic model to test analysis of the above described commercial FRA with four LD pump sources (see Fig. 4). In Fig. 6, the bandwidth of the simulated FRA at $1 \mathrm{~dB}$ level was $50 \mathrm{~nm}$ in the range from 1520 to $1570 \mathrm{~nm}$ and the gain ripple was about $0.5 \mathrm{~dB}$ in the specified bandwidth 1528-1562 nm. The theoretical results based on the proposed model have a good agreement with the numerical data from commercial FRA specifications. Test modeling showed that the FRA ranged from 1528 to $1562 \mathrm{~nm}$ with $1 \mathrm{~dB}$ of the gain flatness could be obtained as seen in Fig. 6.

This $4 \lambda$ amplifier have irremovable by pumping power gain the slope more than $10 \mathrm{~dB}$ in L-band as shown in Fig. 6, and it can not be used in the long wavelength telecommunication window. However, it is known that the pumping power at the long wavelength is usually enhanced by the interaction between short and long wavelength pumping sources, when the distributed
Raman amplifier is in operation. To improve the gain flattening of FRA with the multiwavelength pumping scheme, only about $10 \%$ of pumping power at the long wavelength should be used for amplification.

We proposed to utilize the rest part of the pumping power at the long wavelength for the generation of other pumping sources at long wavelengths based on the Raman lasers with a fiber grating cavity. Using our model, we obtain that the gain bandwidth of FRA can be extended to the L-band, if only two pumping sources at 1486 and $1510 \mathrm{~nm}$ are added. Fig. 7 shows the experimental scheme of FRA with six pumping sources for the extension of the gain bandwidth to L-band. It is composed of 4 pumping $\operatorname{LD}\left(\lambda_{p}=1426,1436,1456\right.$, and $\left.1466 \mathrm{~nm}\right)$ and two grating cavities (1486 and $1510 \mathrm{~nm}$ ) to extend the bandwidth of FRA ranged from the C- to L-band with the small gain ripple. Fig. 8 shows the theoretical results of FRA with the wide bandwidth over $80 \mathrm{~nm}$ and the low gain ripple less than $0.5 \mathrm{~dB}$. The gain ripple can be minimized by controlling the pumping power of each source. The minimal gain ripple of about $<0.5 \mathrm{~dB}$ in full $\mathrm{C}+\mathrm{L}$ bands is achieved when the effective pumping powers is in ratio $0.8: 1.0: 0.75: 0.7: 1.1: 1.5$ for each pumping sources, accordingly.

In addition, the actual band model allows us to estimate the threshold power of Raman lasers used for a pumping source. The threshold power of the pumping source can be achieved, if the input pump power for the pure amplification of the spontaneous Stokes emission becomes more than the fiber loss, $\alpha_{s}$.

It should be noted that the Raman threshold is defined in the early experimental works [15] as the input pump power at which the Stokes power becomes equal to the pump power at the fiber output. Such definition of the SRS threshold was almost exclusively used in the first experimental reports and next for its theoretical description [10], but there is a little bit other definition of the amplification properties of active materials, which is well known as a laser threshold. Next we apply the standard laser threshold definition to the SRS process.

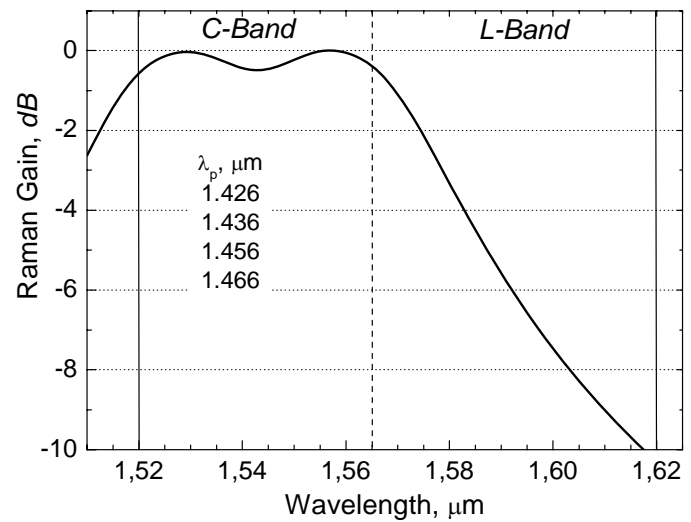

Fig. 6. FRA bandwidth with 4 LD pumping that is simulated based on the actual band model. Amplification slope is more than $10 \mathrm{~dB}$ in L-band.

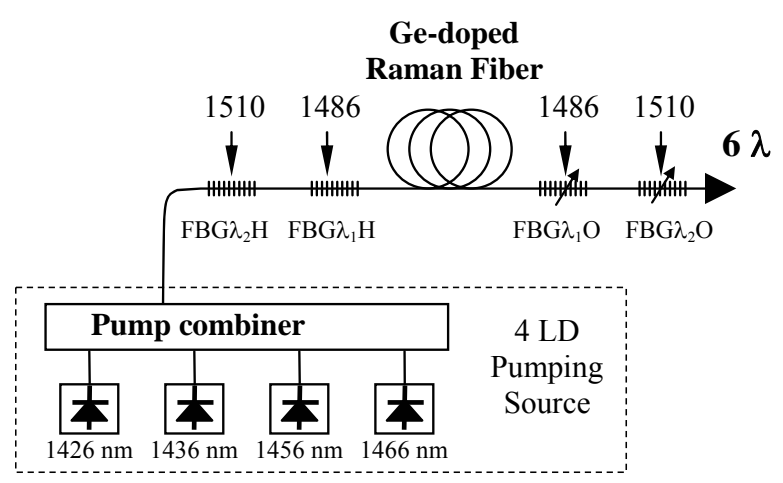

Fig. 7. Experimental setup of $6 \lambda$ pumping source for FRA with the extended bandwidth to L-band. 
The initial growth of the Stokes wave depends on the pump power, and it is described by the Raman-gain coefficient $g_{\mathrm{R}}$. To find the Raman threshold, one should consider the interaction between the pump wave and one monochrome Stokes wave. In the cw case, this interaction is described by the following set of two coupled equations

$$
\begin{aligned}
& \frac{d I_{\mathrm{S}}}{d z}=g_{\mathrm{R}} I_{p} I_{\mathrm{S}}-\alpha_{\mathrm{S}} I_{\mathrm{S}}, \\
& \frac{d I_{p}}{d z}=\frac{\omega_{p}}{\omega_{\mathrm{S}}} g_{\mathrm{R}} I_{p} I_{S}-\alpha_{p} I_{p},
\end{aligned}
$$

where the absorption coefficients $\alpha_{\mathrm{S}}$ and $\alpha_{p}$ account for the fiber loss at the Stokes and pump frequencies, $I_{\mathrm{S}}$ is the Stokes intensity, $I_{p}$ is the pump intensity, and $g_{\mathrm{R}}$ is the Raman-gain coefficient. The Raman-gain coefficient is related to the cross-section of spontaneous Raman scattering, an experimentally measurable quantity. One can readily verify that the laser threshold not only is naturally determined from the standard coupled equations (8)-(9) for the SRS process, but its frequency dependence can be obtained in the quantitative form, being based only on fundamental parameters of the fiber media, i.e., $g_{\mathrm{R}}(\omega)$ and $\alpha(\omega)$.

Really the value of $d I_{\mathrm{S}} / d z$, as it directly follows from the equation (8), can change its sign depending on the pump power. The case of $d I_{\mathrm{S}} / d z<0$ occurs without the pump or at the low pump power and it corresponds to attenuation of the Stokes wave in the course of propagation due to intrinsic losses in the fiber. SRS amplification of the Stokes wave described by product $g_{\mathrm{R}} I_{p}$ is arisen with the growth of pump intensity $I_{p}$, and its numerical value can exceed the meaning of intrinsic losses. In this case, the fiber is transformed into the amplifying media because the condition $d I_{\mathrm{S}} / d z>0$ corresponds to increase of the Stokes wave running in the fiber. In particular case $d I_{\mathrm{S}} / d z=0$, the absolute transparency regime for the Stokes wave propagation in the fiber occurs when the Stokes intensity remains constant along the fiber length.

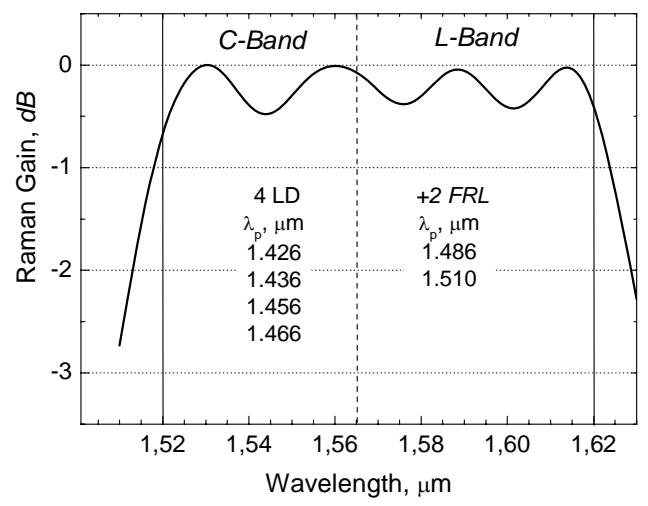

Fig. 8. Simulation of FRA with the large bandwidth over $80 \mathrm{~nm}$ and the low gain ripple $<0.5 \mathrm{~dB}$. Effective pumping powers is in the ratio $0.8: 1.0: 0.75: 0.7: 1.1: 1.5$.
The condition of the fiber absolute transparency in its physical sense corresponds to the laser threshold of the SRS process. The ratio $d I_{\mathrm{S}} / d z=0$ together with the equation (8) gives the quantitative expression for the limiting condition when the material of fiber core starts to transform from the natural state with attenuation of the Stokes wave to the state in which the Stokes wave is amplified due to the pump power. In contrast with experimental threshold, the laser threshold is caused only by material parameters of the fiber, and it does not depend on experimental conditions, in particular it is independent from the fiber length.

Hence, one can directly calculate the laser threshold of the SRS as a function of the Stokes frequency shift (or wavelengths) using the experimental data of the Raman gain profile $g_{\mathrm{R}}(\omega)$ and the known values of fiber losses $\alpha$ for the arbitrary wavelength of the pump source. The gain profile $g_{R}(\omega)$ for Raman fibers is satisfactory described using the simplified actual band approximations as we showed above, whereas it is necessary to use a more complex but exact multimode decomposition model $[16,17]$ of the SRS spectrum for other fibers.

It should be noted that intrinsic losses at the Stokes frequency are $\alpha \sim 0.2 \mathrm{~dB} / \mathrm{km}$ only for pure silica fibers. Substantial increasing the Raman gain in fiber is achieved due to the raised doping concentration of $\mathrm{GeO}_{2}$ (typically $\sim 20 \%$ ) when manufacturing these Raman fibers, but this process, unfortunately, is accompanied by appreciable increase of intrinsic fiber losses with minimal values $\alpha \sim 0.3 \ldots 1.0 \mathrm{~dB} / \mathrm{km}$. Therefore, in Fig. 9 resulted are the laser thresholds of the SRS $\left(\lambda_{p}=\right.$ $=1.45 \mu \mathrm{m})$ as a function of the wavelength for the Raman fibers with various values of intrinsic losses, which change in the range from 0.2 up to $1.0 \mathrm{~dB} / \mathrm{km}$. The threshold power for the monochrome Stokes signal at $\lambda_{\mathrm{S}}=1.55 \mu \mathrm{m}$ naturally grows from approximately 9 up to $40 \mathrm{~mW}$ when the intrinsic losses are increased from 0.2 up to $1.0 \mathrm{~dB} / \mathrm{km}$. If the value of the pump power is below the laser threshold, i.e., laid in the

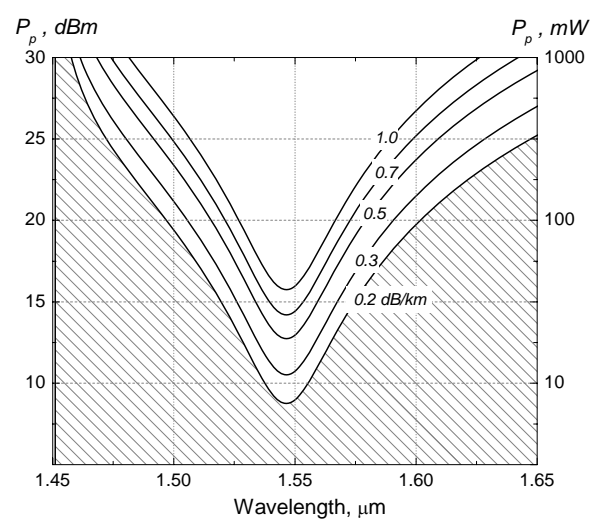

Fig. 9. SRS laser threshold $\left(\lambda_{p}=1.45 \mu \mathrm{m}\right)$ in Raman fibers with various intrinsic losses. Stokes waves have attenuation in the shaded under area curves and the SRS amplification is realized in light area above curves. 
shaded area of Fig. 9, than the Stokes waves have attenuation, and SRS amplification is realized in the light area above curves.

Our resulting calculations show that the laser threshold for the monochrome Stokes signal takes place in a sufficiently wide spectral range when the pump power is raised up to several hundreds milliwatts irrespectively of real fiber losses. In this case, the laser generation can be obtained in the range approximately from 1.5 up to $1.6 \mu \mathrm{m}$ at the pump wavelength $\lambda_{\mathrm{p}}=1.45 \mu \mathrm{m}$, that is in the whole band of maximal fiber transparency. It is necessary to emphasize that the spectral position of narrowband laser generation can be shifted by simple changing the pump wavelength. Thus, the absolute values of the threshold power can be calculated when one takes into account the frequency dependences of main fiber parameters such as the SRS gain coefficient $g_{\mathrm{R}}(\omega)$ and fiber loss parameter $\alpha(\omega)$.

These theoretical estimations prove to be true by the numerous experimental results (see, for example [18]) of the last time. Finally, we apply the proposed model to determine lasing threshold pump powers for each LD in the arrangement of Fig. 7. We assume that $P_{p}(\omega)$ can be found when the pump wave interacts with the spontaneous Stokes one, and it generates the stimulated power $P_{\mathrm{S}}(\omega)$. After substitution of the pump intensity as $I_{p}(\omega)=P_{p}(\omega) / A_{\text {eff }}$ in (8), one can easy verify that travelling Stokes wave in $z$-direction of fiber begins to increase $\left(d P_{\mathrm{S}}(\omega) / d z>0\right)$ under the condition of

$P_{p}(\omega) \geq \frac{\alpha_{\mathrm{S}} A_{\mathrm{eff}}}{g_{r}(\omega)}$.
Since the proposed spectroscopic model can give the function $g_{\mathrm{R}}(\omega)$, we can get the threshold power $P_{p}^{\mathrm{thr}}(\omega)=\alpha_{\mathrm{S}} A_{\mathrm{eff}} / g_{r}(\omega)$ of pumping source to get a gain.

Figs $10 \mathrm{a}$ and $\mathrm{b}$ shows the threshold power of each pump source under the fiber loss of $0.2 \mathrm{~dB} / \mathrm{km}$ and the threshold power at $1426 \mathrm{~nm}$ for various fiber losses, respectively. The detailed parameters of each pumping source for Raman generation of two additional pumping sources based on grating cavities are shown in Table.

Table. Threshold powers for Raman lasing at $\lambda_{1}$ with pumping sources on $\lambda_{p}$.

\begin{tabular}{|c|c|c|}
\hline \multirow{2}{*}{$\begin{array}{c}\text { Pumping } \lambda_{p}, \\
\mu \mathrm{m}\end{array}$} & \multicolumn{2}{|c|}{$\begin{array}{c}\text { Threshold power in } \mathrm{mW} \text { for Raman } \\
\text { lasing at } \lambda_{l}, \mu \mathrm{m}\end{array}$} \\
\cline { 2 - 3 } & 1.486 & 1.510 \\
\hline 1.426 & 42.6 & 9.7 \\
\hline 1.436 & 69.6 & 20.3 \\
\hline 1.456 & 144.0 & 62.7 \\
\hline 1.466 & 190.9 & 93.9 \\
\hline
\end{tabular}

It was noted that we could use the actual band model with a good conformity for the analysis of practical FRA in a Ge-doped fiber. Using the proposed model, we designed the FRA scheme with the broad bandwidth in both C- and L-band based on the Raman lasing in fibers. We could obtain the practical results with the proposed modeling, which contained the key FBG parameters and lasing thresholds. (a)

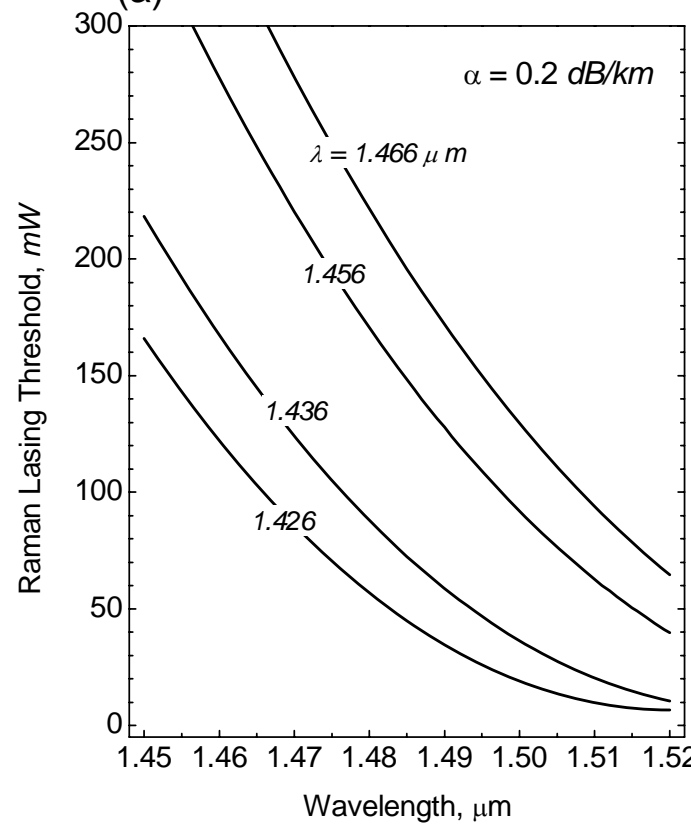

(b)

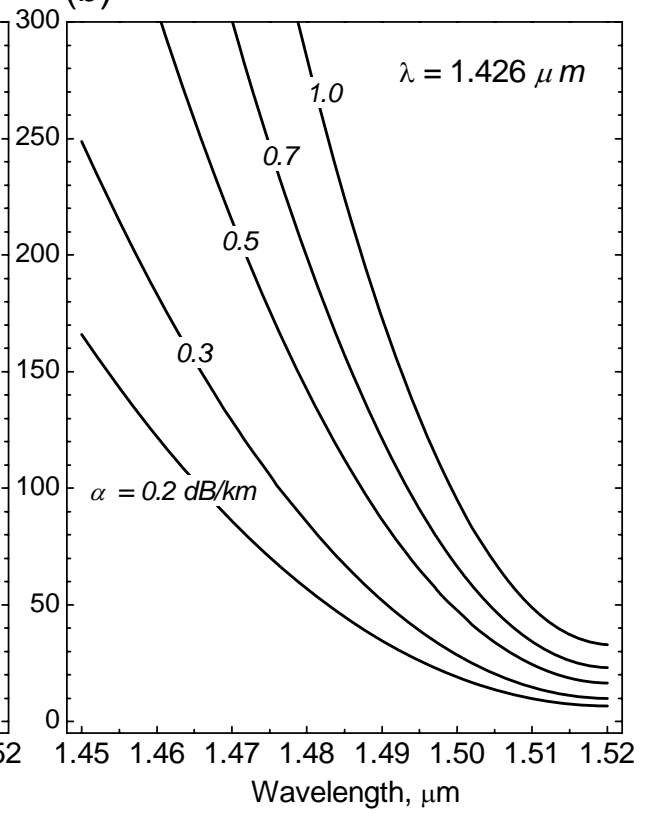

Fig. 10. Threshold pumping power for Raman lasing with respect to different pumping wavelength (a) and different optical loss in Ge-doped fibers (b). $g_{\max }\left(\operatorname{Raman}\right.$ fiber $\left.\mathrm{GeO}_{2}\right)=6.1(\mathrm{~W} \cdot \mathrm{km})^{-1}[12]$. 


\section{Conclusion}

In summary, the spectroscopic model for the design of FRA with the multiwavelength-pumping scheme based on the theory of the Raman gain coefficient of an optical fiber was investigated. The oscillatory lineshape functions of $S_{\mathrm{R}}(v)$ can simply describe the wavelength dependence of the Raman gain in optical fibers and are very useful for the estimation of the gain bandwidth, the Raman lasing, noise performance, and amplification processes in Raman amplifiers. The concept of an actual band based on the lineshape function $S_{\mathrm{R}}(v)$ is useful for the design of FRA with multiple wavelength pumping.

The carried out measurements of ASE cross-section show that the output FRA optical noise is corresponded to quantum efficiency of the spontaneous Raman scattering, but it does not SRS. The numerical values for ASE crosssection are almost the constant. The Raman quantum efficiency grows no more than $\sim 40 \%$ when the pump powers are 3 times raised. We obtained the greatest possible quantities for the ASE on/off gain coefficients are about $1.9 \mathrm{~dB}$ in the $50 \mathrm{~km}$ span of single mode silica fiber. Thus the physical principles of formation of the FRA optical noise with the counter pumping almost correspond to the spontaneous Raman properties in the optical fibers based on fused silica. Accordingly, one can use the proposed actual band model for quantitative determining the power spectrum of output FRA optical noise. It is sufficient to know the pump power distribution at the amplifier input in this modeling procedure.

As practical results of our modeling, we obtained the parameters of FBG and lasing thresholds based on the actual band model with the good conformity for the analysis of experimental set up parameters for FRA in Ge-doped fibers. In addition, application of our model allows us to get the information about the nature of the noise parameter formation mechanism in the practical FRA. The proposed spectroscopic model can be further extended for the analysis of the complex SRS spectra with other doping materials.

\section{References}

1. S.K. Kim, S.H. Chang, J.S. Han, M.J. Chu, and J.H. Lee, Distributed fiber Raman amplifiers with localized loss // J. Lightwave Techn. 21(5), p. 12861293 (2003).

2. M.N. Islam, Raman amplifiers for telecommunications // IEEE J. Sel. Top. Quantum Electron. 8(3), p. 548-559 (2002).

3. V.E. Perlin, and H.G. Winful, On distributed Raman amplification for ultrabroad-band long-haul WDM systems // J. Lightwave Techn. 20(3), p. 409-416 (2002).

4. H. Kidorf, K. Rottwitt, M. Nissov, M. Ma, and E. Rabarijaona, Pump interactions in $100 \mathrm{~nm}$ bandwidth Raman amplifier // IEEE Photon. Technol. Lett. 11(5), p. 530-532 (1999).
5. M. Yan, J. Chen, W. Jiang, J. Li, J. Chen, and X. Li, Automatic design scheme for optical-fiber Raman amplifiers backward-pumped with multiple laser diode pumps // Ibid. 13(9), p. 948-950 (2001).

6. P. Xiao, Q. Zeng, J. Huang, and J. Liu, A new optimal algorithm for multipump sources of distributed fiber Raman amplifier // Ibid. 15(2), p. 206-208 (2003).

7. I. Mandelbaum, and M. Bolshtyansky, Raman amplifier model in single-model optical fiber // Ibid. 15(12), p. 1704-1706 (2003).

8. K. Rottwitt, J. Bromage, A. J. Stentz, L. Leng, M.E. Lines, and H. Smith, Scaling of the Raman gain coefficient: Applications to germanosilicate fibers // J. Lightwave Techn. 21(7), p. 1652-1662 (2003).

9. M.L. Dakss and P. Melman, Amplified spontaneous Raman scattering and gain in fiber Raman amplifiers // J. Lightwave Technol. LT-3(4), p. 806-813 (1985).

10. G.P. Agrawal, Nonlinear fiber optics, $2^{\text {nd }}$ ed. Academic, San Diego, CA, 1995.

11. R. Loudon, The quantum theory of light, $2^{\text {nd }}$ ed. Clarendon Press, Oxford, 1983.

12. S.T. Davey, D.L. Williams, and B.J. Ainslie, Optical gain spectrum of $\mathrm{GeO}_{2}-\mathrm{SiO}_{2}$ Raman fiber amplifiers // Proc. Inst. Elect. Eng. 136(6), p. 301305 (1989).

13. M.D. Mermelstein, C. Horn, S. Radic and C. Headley, Six-wavelength Raman fiber laser for C-and Lband Raman amplification and dynamic gain flattening // Electron. Lett. 38(13), p. 636-638 (2002).

14. J. Bromage, K. Rottwitt, and M.E. Lines, A method to predict the Raman gain spectra of germanosilicate fibers with arbitrary index profiles // IEEE Photon. Technol. Lett. 14(1), p. 24-26 (2002).

15. R.H. Stolen, C. Lee, and R.K. Jain, Development of the stimulated Raman spectrum in single-mode silica fibers // J. Opt. Soc. Amer. B 1(4), p. 652-657 (1984).

16. G.S. Felinskyi, Spectroscopic multiple-vibrationalmodeling of Raman gain for FRA design // Proc. $7^{\text {th }}$ Intern. Conf. on Laser and Fiber-Optical Networks Modeling (LFNM 2005), September 12 17, 2005, Yalta, Crimea, Ukraine, p. 262-265.

17. G.S. Felinskyi, P.A. Korotkov, Actual band model for design of optical fiber Raman amplifier with multiwave pumping // Proc. $2^{\text {nd }}$ Intern. Conf. on Advanced Optoelectronics and Lasers (CAOL 2005), September 12-17, 2005, Yalta, Crimea, Ukraine, 2, p. 168-171.

18. E.M. Dianov, A.M. Prokhorov, Medium-power cw Raman fiber lasers // IEEE J. Sel. Top. Quantum Electr. 6(6), p. 1022-1028 (2000). 